\title{
Pratiques
}

Linguistique, littérature, didactique

183-184| 2019

oralité, littératie

\section{Oralité et littératie dans l'enseignement professionnel}

Orality and literaty in agricultural education: a challenge in terms of socioeducational inequalities.

\section{Laure Minassian}

\section{(2) OpenEdition}

\section{Journals}

Édition électronique

URL : http://journals.openedition.org/pratiques/7602

DOI : $10.4000 /$ pratiques.7602

ISSN : 2425-2042

Éditeur

Centre de recherche sur les médiations (CREM)

Référence électronique

Laure Minassian, « Oralité et littératie dans l'enseignement professionnel », Pratiques [En ligne], 183-184 | 2019, mis en ligne le 30 décembre 2019, consulté le 09 octobre 2020. URL : http:// journals.openedition.org/pratiques/7602 ; DOI : https://doi.org/10.4000/pratiques.7602

Ce document a été généré automatiquement le 9 octobre 2020.

(c) Tous droits réservés 


\title{
Oralité et littératie dans l'enseignement professionnel
}

\author{
Orality and literaty in agricultural education: a challenge in terms of socio- \\ educational inequalities.
}

Laure Minassian

\section{Introduction}

1 Comment s'enseigne un domaine où s'interpénètrent oralité et littératie tel que celui de l'agriculture? Quelles sont les pratiques enseignantes à l'articulation des sphères scolaire et professionnelle, lesquelles donnent lieu à des événements, des pratiques sociales des valeurs différents? Un des apports de l'article consiste à montrer dans quelle mesure l'école transforme ce qui est fait avec l'oralité et la littératie dans le cadre du travail pour en faire un objet scolaire.

2 Sur un versant anthropologique la littératie s'étend à l'ensemble des activités humaines qui recourent à l'écriture et en retour contribuent à structurer la pensée. Le rapport entre sociétés et écriture a été mis en avant par l'anthropologue J. Goody, montrant combien cette médiation a permis de mémoriser, catégoriser le monde et à le communiquer (Goody, 1979 [1977]). Toutefois, S. Scribner et M. Cole (1973) - en suivant trois groupes d'Afrique occidentale respectivement scolarisés en anglais, en arabe et non scolarisés - ont montré qu'au plan individuel la maitrise de l'écrit ne donne pas toujours lieu au développement prévu de la pensée, nuançant ainsi les effets cognitifs directs de celui-ci. Autrement dit, l'appropriation du sens est toujours hétérogène et partielle. Elle est la résultante de processus d'interactions entre un contexte, des ressources symboliques comprenant de l'écrit et un éventail de dispositions des sujets (Heath \& Street, 2008).

3 Si nous souscrivons à cette définition, à savoir que deux élèves placés devant le même écrit peuvent mettre en œuvre des pratiques de sens socio-culturellement et différentiellement situées, notre objectif dans le cadre de cette contribution est de 
donner à voir en quoi les écrits, les consignes, les tâches, à effectuer dans le cadre scolaire réfèrent plutôt à l'oralité ou la littératie, indépendamment de ce qu'en font les élèves. Ces éléments sont d'importance car écrit et oral ne s'opposent pas tout à fait. Un texte peut s'inscrire dans l'oralité et un discours peut être hautement littératié. Ce qui nous intéresse est de porter la focale sur des indices fins (notamment sémiotiques et syntaxiques) qui renvoient de façon dominante, mais non exclusive à un univers littératié ou d'oralité - car la littératie est toujours emprunte d'oralité et inversement. Nous reprenons à M. Laparra et C. Margolinas la distinction entre ces notions, c'est-àdire que :

nous disons qu'il y a oralité quand un groupe humain - ici le groupe classe pratique des échanges verbaux ou non, sur et à l'aide d'objets du monde, en mettant en jeu les ressources corporelles dont il dispose, de manière fortement routinisée. Nous disons qu'il y a littératie quand un groupe humain se meut et agit dans un espace qui organise les corps et les objets du monde selon les ressources fournies par l'écrit et qu'il pratique un usage raisonné et organisé de ces ressources». (Laparra \& Margolinas, 2012, p. 57).

4 Le propos est développé en trois temps. En premier lieu, une analyse comparative des contextes éclaire des différences entre les deux établissements d'enquête et leurs publics; ensuite l'analyse de l'activité pédagogique porte sur les concepts, objets et méthodes permettant, enfin, d'objectiver les compositions des supports de cours et les univers convoqués. Ces quelques éléments permettront de discuter ces pratiques et leurs effets potentiels.

\section{1 - Des contextes d'enseignement et des publics}

5 Loin de correspondre à une dichotomie formel/informel, certains écrits sont peu formalisés (notes, cahiers, brouillons...) alors que certaines communications orales le sont plus (exposé, réponse ponctuelle, ébauche de définition...; Grosjean \& Lacoste, 1998, p. 449). En outre, l'écrit est certes un objet privilégié de l'école, voire en est constitutif, mais il l'est également au travail (Taylor, 1983) au travers de «cahiers personnels, feuilles de route, petits carnets, etc. » (Boutet, 2008).

$6 \mathrm{Au}$ moins parce que les élèves fréquentent un certain milieu professionnel, la dimension du comment les écrits constituent des objets de transaction, de traduction entre mondes sociaux distincts et parfois distants (Latour \& Woolgar, 1988 [1986]) a retenu notre attention.

7 Cette attention est aussi orientée par la spécificité des écoles observées: un lycée académique des Frères de l'Instruction Chrétienne de Ploërmel et une Maison Familiale Rurale (MFR).

8 Les données présentées proviennent d'une enquête ethnographique comportant une présence de six mois dans chacune des classes observées, accompagnées d'enregistrements d'entretiens audio et vidéo des cours, ainsi que d'un recueil pendant deux années de dossiers à forte composante technique constitués par les élèves dans le cadre du contrôle continu. Dans le cadre de cette contribution, l'analyse est centrée sur les supports de cours.

9 Les écoles choisies sont relativement typées en fonction de leur distance/proximité au regard de la forme scolaire traditionnelle. Leur distance différentielle fait écho, encore aujourd'hui, à la constitution historique de ces écoles. Construit au XIXe siècle, le lycée a 
longtemps fondé sa pédagogie d'inspiration lassalienne sur la séparation des sphères domestique et scolaire tandis que l'enseignement en MFR a été pensé, dès sa création en 1935, comme un lieu de continuité entre ces sphères. Si, sur le plan de l'offre de formation, ces écoles sont aujourd'hui unifiées et dispensent les mêmes standards de diplômes, les diplômes inférieurs au baccalauréat sont plus importants en MFR tandis que les élèves de terminales scientifiques et des BTSA (Brevet de technicien supérieur agricole) sont proportionnellement plus nombreux dans les lycées (Minassian, 2015). Ce niveau de diplôme a fait l'objet de l'analyse dans la spécialité Conduite et Gestion d'une Exploitation Agricole parce qu'il constitue le «sésame» pour s'installer en tant qu'exploitant agricole.

10 Cette graduation tend à se transmuer en opposition lorsque sont considérés les publics accueillis, bien qu'ils soient de manière écrasante d'origine populaire de milieu rural mais aussi de petites communes, et, plus rarement de résidence périurbaine. Comme à l'échelle nationale (Minassian, 2015), les élèves socialement éloignés du milieu agricole (souvent fils d'ouvriers et/ou d'employés) et plutôt en difficultés scolaires, s'orientent dans la MFR où le temps de stage est le plus long ( $50 \%$ du temps pédagogique) tandis que les élèves originaires du milieu agricole davantage en proximité avec les attentes de l'école s'inscrivent dans l'établissement où le temps de stage est plus court ( $25 \% \mathrm{du}$ temps pédagogique). Une des raisons relève de leur parcours antérieur. Alors que les élèves de MFR proviennent pour les deux-tiers d'une classe de troisième technologique ou professionnelle, la plupart des élèves de la classe du lycée proviennent d'une troisième générale. Souvent confrontés à l'échec scolaire, les récits des élèves de MFR sur leurs parcours sont ponctués de tentatives pour réussir, de résignation, de mises à l'écart.

11 Un des faits à souligner est qu'ils ont tendance à matérialiser leur rapport à la littératie, pour en faire des objets du monde et non des objets pour penser. Les expressions suivantes sont courantes en entretien: " à l'école on n'fait rien d'ses mains, à part écrire ", "c'est qu'du papier ", "l'école c'est du travail sur feuille ", "c'est qu'des chiffres d'toute façon ».

De façon contrastée, au lycée, les élèves ont développé des justifications internes à la culture scolaire : «en maths j'ai toujours été bon », « cette année on étudie la poésie et le théâtre... ben le théâtre j'aime bien ça », « les verbes... où est-ce qu'ils sont... quelles sont leurs valeurs ", « tout le français j'aime bien ", « ce que j'préfère c'est les dissert ». Cet appétit scolaire se retrouve dans les domaines qu'ils valorisent : le dessin, les arts plastiques, le théâtre, la littérature et la philosophie, la politique.

$13 \mathrm{Vu}$ de loin, les écoles de l'enseignement professionnel paraissent proches, mais le cas décrit montre que dès lors qu'on approche la focale, l'enseignement professionnel se montre composite et pluriel. L'intérêt d'entrer dans la classe et d'analyser les objets pour apprendre devient un sérieux indicateur de ce que l'école fabrique avec ces publics forts différents.

\section{2 - Littératie, oralité : de la classe à l'établissement}

\section{A. Le statut des supports de cours et d'exercice}

14 Une des particularités de l'enseignement professionnel, comme de l'enseignement agricole, est qu'il est peu doté en manuels scolaires. Les supports d'enseignement sont 
élaborés par les enseignants à la suite d'interprétations des référentiels de métier et de formation nationaux en réunion d'équipe. En ce sens, ces supports peuvent être considérés comme un témoignage du « genre professionnel » de l'établissement, car la configuration de leur élaboration porte des traces des valeurs implicites que partagent ceux qui y travaillent (Clot, 2008). Bien sûr, ces supports portent aussi le style de l'enseignant en ce qu'il recrée au singulier un usage propre du genre. Reste que la configuration très particulière des contextes dans lesquels s'élaborent et prennent place les supports, amène à souligner que les analyses ici produites s'étendent au-delà de la classe et interrogent plutôt l'établissement tout entier, voire l'institution.

\section{B. Les choix pour l'analyse}

15 Les manières de choisir et d'ordonner des éléments dans les supports participe de la convocation d'un univers où domine de façon plus ou moins prégnante la littératie ou l'oralité. L'analyse porte donc sur les contenus tout autant que sur un ensemble de procédés sémiotiques qui traversent ces documents tels que les types de mise en page. Ces éléments donnent à voir des construits sociaux qui se traduisent par la valorisation de certains éléments au détriment d'autres, au travers de listes par puces, mises en retrait, paragraphes continus, tableaux, encadrés, signes graphiques, titres, soulignements, types de caractères, etc. La mise au premier plan des éléments présentés comme les plus pertinents, les formes linguistiques que prennent les consignes, en particulier les formes verbales et averbales, les formes de consignes et les types d'écrit attendus que supposent ces consignes (argumentatif, descriptif, liste d'indicateurs par exemple) sont autant d'indices à prendre en compte dans l'analyse.

\section{Les thèmes d'apprentissage à l'école d'agriculture}

Le thème du cours analysé concerne l'alimentation des vaches laitières au regard de leur production. Ce thème a été choisi car il est le plus spécifique et le plus traditionnel de la discipline enseignée (Denis \& Théret, 1994). Les phénomènes liés à l'alimentation sont dépendants de trois éléments : la production laitière, la taille et poids de l'animal et enfin la qualité du fourrage. Le processus métabolique de l'animal pour produire du lait est le produit d'un rapport entre les protéines issues de la cellulose (donc de la fibre qui dépend de la qualité du fourrage) et les protéines digestibles sous forme d'énergie (que l'on trouve en grande quantité dans le blé, le soja, le tournesol, etc.). C'est ce rapport qu'il faut équilibrer par l'intermédiaire de calculs (au regard du poids et de la production laitière de l'animal). Ces principes peuvent être mis en relation avec des savoirs zoologiques, (de quoi dépend la capacité d'ingestion d'une vache, quels sont les risques métaboliques de l'introduction de types d'aliments, sont des composantes typiques de la science appliquée à l'élevage). Ils peuvent aussi entrer dans une seconde logique davantage en lien avec l'efficacité de l'action, la performance technique. Il en va ainsi des éléments d'anticipation : la production fourragère permet-elle de nourrir les animaux une année? Sur quelle parcelle implanter quel type de fourrage? Quel type d'assolement faut-il prévoir? Ces deux types de logiques possibles ne sont pas de même nature, elles ne sollicitent pas les élèves de la même manière et orientent des rapports à la production différents. 


\section{Les données de l'analyse}

Dans les classes observées, un des premiers constats relève du nombre et de la diversité des supports pédagogiques. En effet, alors qu'en MFR, il existe un seul et même type de support pour l'ensemble des thèmes de cours abordés (alimentation du troupeau, santé des animaux, qualité du lait, etc.), il en existe quatre au lycée agricole à raison d'un pour chacun de ces thèmes. Ils peuvent être ordonnés sous les catégories suivantes :

- cours magistral (supports pédagogiques de type 1);

- devoirs sur table (supports pédagogiques de type 2) ;

- travaux dirigés (supports pédagogiques de type 3);

- consignes pour la construction des dossiers : études techniques d'exploitation (supports pédagogiques de type 4 ).

Cette disparité importante n'est pas sans rappeler cette ancienne distinction entre le cahier du jour dans l'enseignement primaire et le classeur dans le secondaire (Chartier \& Renard, 2000).

Dans le cadre de cette étude, les documents de type 4 du lycée ont été retenus par souci de comparaison avec les documents de MFR. En effet, ces deux types de documents ont en commun de porter des consignes pour la rédaction de dossiers techniques écrits par les élèves au sujet des exploitations agricoles.

Tableau 1. Type de support et d'activité scolaire dans les deux classes observées

\begin{tabular}{|c|c|c|c|}
\hline \multicolumn{2}{|c|}{$\begin{array}{l}\text { Classe à supports différenciés } \\
\text { (Lycée agricole) }\end{array}$} & \multicolumn{2}{|c|}{$\begin{array}{l}\text { Classe à supports indifférenciés } \\
\text { (MFR) }\end{array}$} \\
\hline Supports d'activité scolaire & Type d'activité & Supports d'activité scolaire & Type d'activité \\
\hline Type 1 & Apports notionnels & Un seul type de support & \\
\hline $\begin{array}{l}\text { Type } 2 \\
\end{array}$ & Devoirs sur table & & \\
\hline Type 3 & $\begin{array}{l}\text { Inventaire des documents à } \\
\text { rechercher sur le lieu de } \\
\text { stage }\end{array}$ & & \\
\hline Type 4 & $\begin{array}{l}\text { Consignes pour la } \\
\text { réalisation du dossier de } \\
\text { stage }\end{array}$ & & $\begin{array}{l}\text { Inventaire des documents à } \\
\text { rechercher sur le lieu de } \\
\text { stage et consignes pour la }\end{array}$ \\
\hline & & & stage \\
\hline
\end{tabular}

\section{3 - Études de cas : agir avec les objets du monde pour agir versus organiser la réflexion dans et par l'écrit}

\section{A. Les supports de la MFR. Des ressources ancrées dans l'oralité pour initier à la prise de décision}

Les supports produits dans la MFR relèvent tout d'abord d'un registre fonctionnel supposant une expérience du métier. Chaque support pédagogique destiné aux élèves est l'occasion de visiter un ensemble d'entités qui composent une exploitation agricole. 
Le support correspondant au chapitre de l'alimentation des vaches laitières participe de cette tendance, et comprend un ensemble de domaines à explorer (voir extrait 1).

L'organisation générale est réalisée par sous-thèmes. En ce qui concerne l'analyse de l'alimentation du troupeau laitier, ces sous-thèmes sont au nombre de trois. Le premier est relatif à l'organisation globale de l'exploitation.

\section{Extrait 1 : L'organisation de l'exploitation}

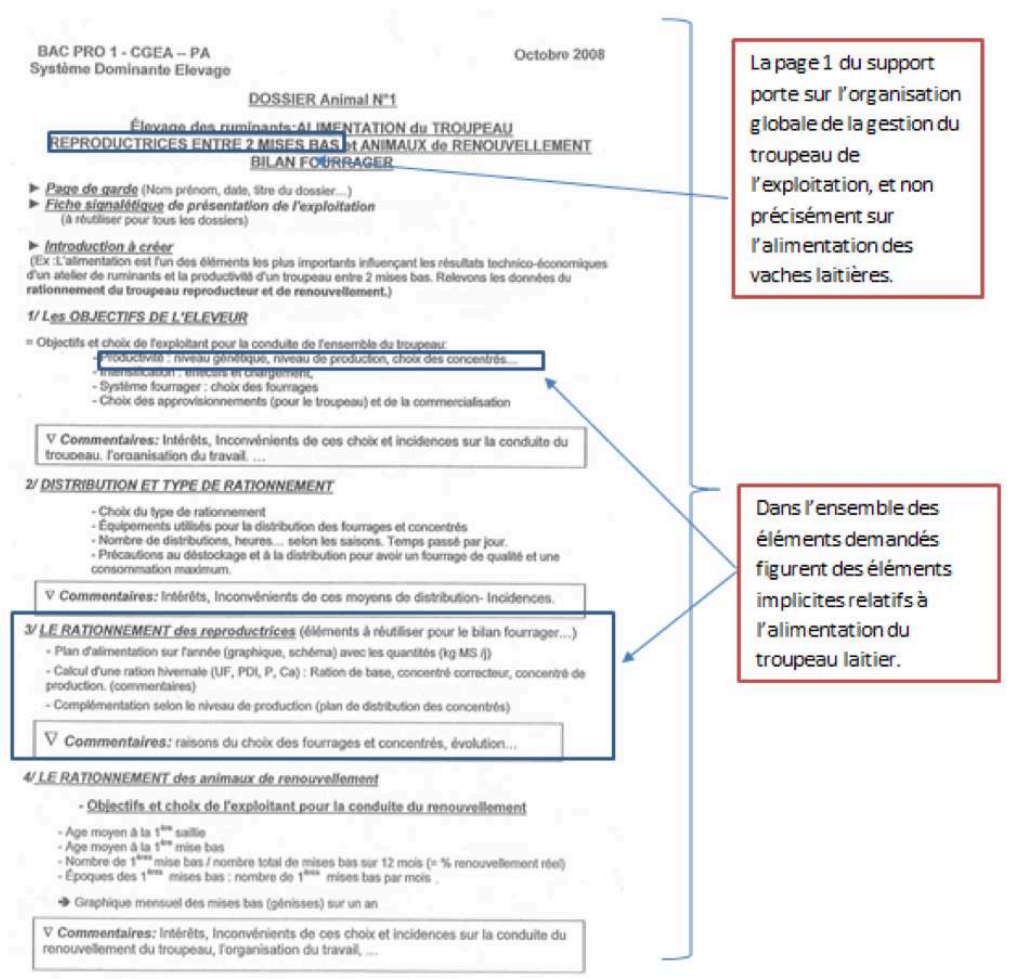

Dans l'extrait ci-dessus, il s'agit de prendre en compte l'exploitation dans son ensemble, et non le troupeau laitier seulement. Sur l'extrait ci-après figure un exercice de calcul des stocks de fourrages disponibles sur la totalité de l'exploitation (par cubage des silos, mais aussi par pesée géométrique). La procédure de calcul du fourrage est donnée de telle sorte que les élèves mesurent le stock existant en unités cubiques, ce qui est particulièrement complexe. Autrement dit, c'est moins un exercice scolaire, au sens du référentiel, "acquérir une méthode d'analyse systémique » (Référentiel de formation), que d'acquérir des formes de raisonnements localement situées comme dans l'exercice du travail. 


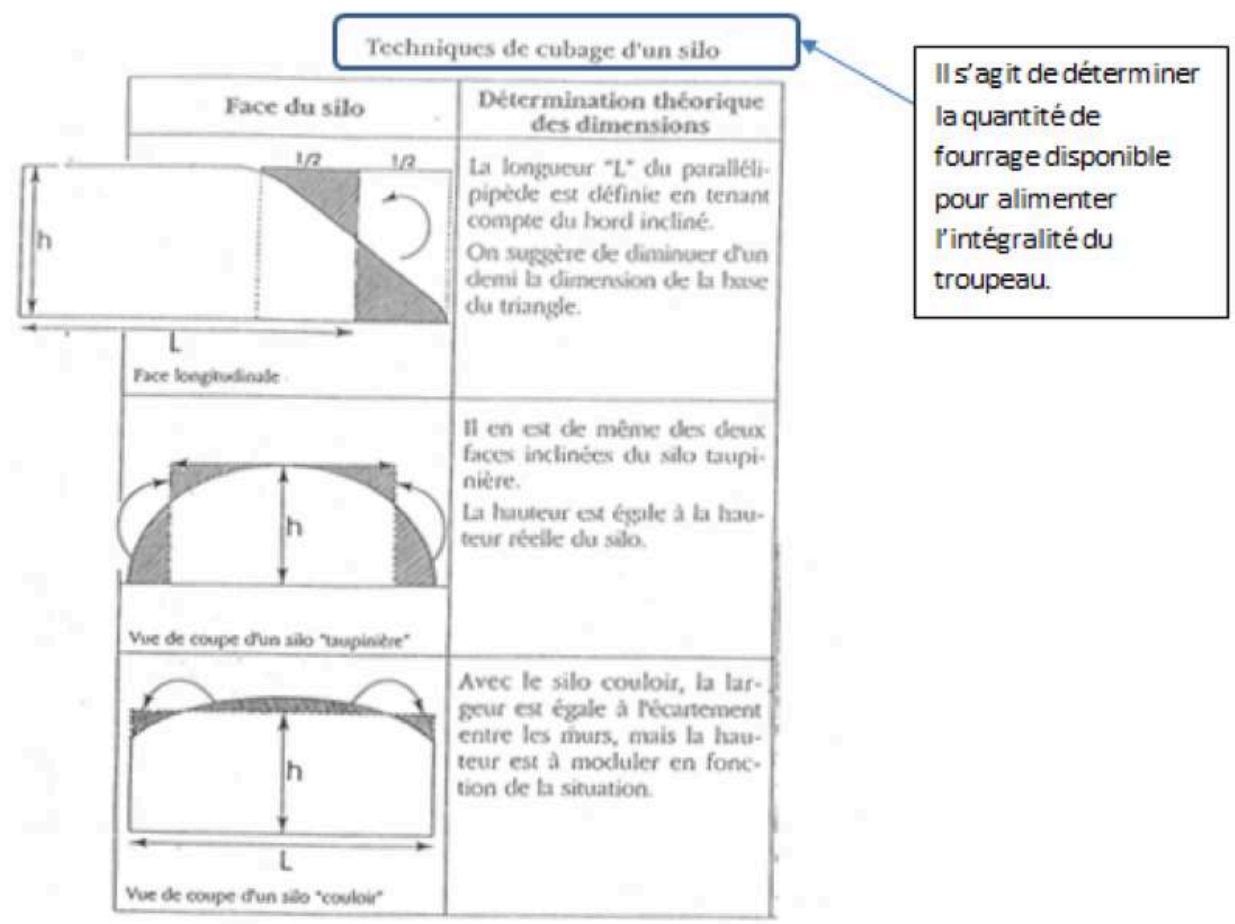

Enfin, la dernière page figure un plan de rationnement alimentaire prévisionnel vierge à compléter où les stocks calculés sont comparés aux stocks consommés sous la forme d'un tableau de bilan fourrager. Ce dernier sous-thème clôt la construction logique de l'ensemble des documents. Il constitue en réalité le principal objectif. Il s'agit d'évaluer des stocks d'aliments au regard de ce que les animaux consomment. Les supports se lisent à partir de l'objectif général, fixé tardivement en dernière page (et non progressivement sous-thème par sous-thème, c'est-à-dire de façon littératiée), en lien avec des préoccupations pragmatiques que l'on peut formuler ainsi : l'exploitation dispose-t-elle de fourrages en quantité suffisante pour tenir une année ? Les besoins et les stocks sont-ils à l'équilibre, excédentaires, déficitaires?

Cette organisation est davantage liée à une configuration dans laquelle il faut faire apparaître les objets du monde d'une certaine manière pour prendre des décisions. Elle n'est en revanche que très peu liée à une activité de catégorisation, de classement, et si elle l'est, les savoirs littératiens à mettre en œuvre restent complétement transparents. Autrement dit, oralité et littératie s'enchâssent dans la perspective du travail de gestion d'un exploitant. En effet, la construction cognitive attendue (prendre en compte un ensemble d'indicateurs pour prendre une décision) n'est jamais pleinement consignée dans l'écrit. Elle repose exclusivement sur l'interprétation du tableau final, qui dépend d'une acculturation aux pratiques sociales des exploitants (avoir assez de stocks en particulier pour l'hiver) pour créer ce sens comme dans l'ensemble des autres supports de cours. La transposition de la pratique professionnelle au contexte scolaire est donc présupposée, elle s'appuie sur le principe qu'il existe chez les élèves une préconnaissance des contextes culturels suffisantes pour que cette transposition aille de soi (Barton \& Hamilton, 1998 ; 2010). 


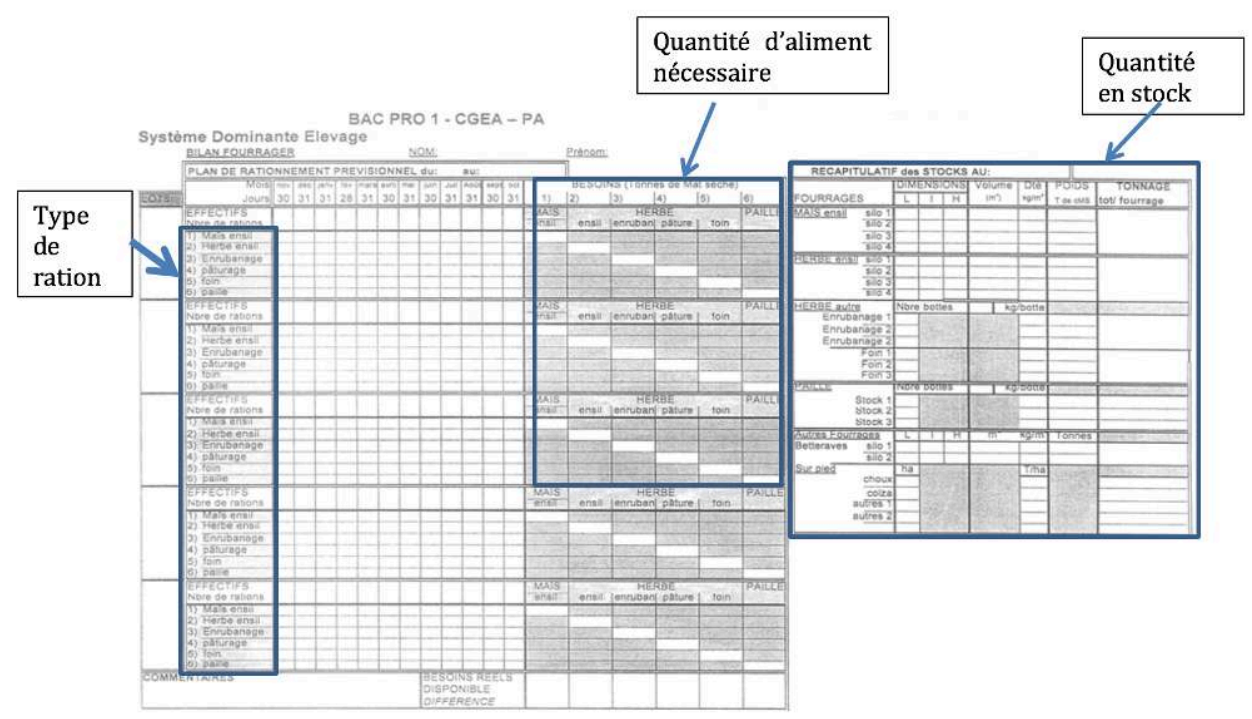

\section{B. Supports du lycée. Des ressources ancrées dans la littératie pour initier une démarche de réflexion}

Les supports du lycée sont caractérisés par le découpage d'une démarche de raisonnement, laquelle prend la forme d'une séquence continue entre différents types de ressources cognitives à mobiliser par les élèves: recherche d'informations sur l'exploitation de stage-comparaisons avec d'autres résultats d'exploitationsinterprétations des écarts-résultats, c'est-à-dire de construire des constats et des analyses grâce à une démarche transposable et reproductible. Le support est organisé en deux grandes parties : la première relève du contexte général de l'exploitation; la seconde, plus longue, est centrée sur la notion de l'alimentation du troupeau laitier. Autrement dit, le thème principal d'apprentissage est rendu plus visible. Dans cette seconde partie, les élèves sont incités à entrer dans une activité de comparaison des valeurs alimentaires des fourrages (le calcul de ces valeurs par exploitation est souvent réalisé par un service de la Chambre d'Agriculture) et de les mettre en regard avec les pratiques culturales des exploitants. Il s'agit de produire à partir d'un exercice, où n'entre pas toute la complexité du réel, un texte argumenté en s'appuyant sur des documents de la Chambre d'Agriculture (des valeurs alimentaires faibles pouvant s'expliquer par les contraintes d'environnement). 
Extrait 4. Un support en deux parties

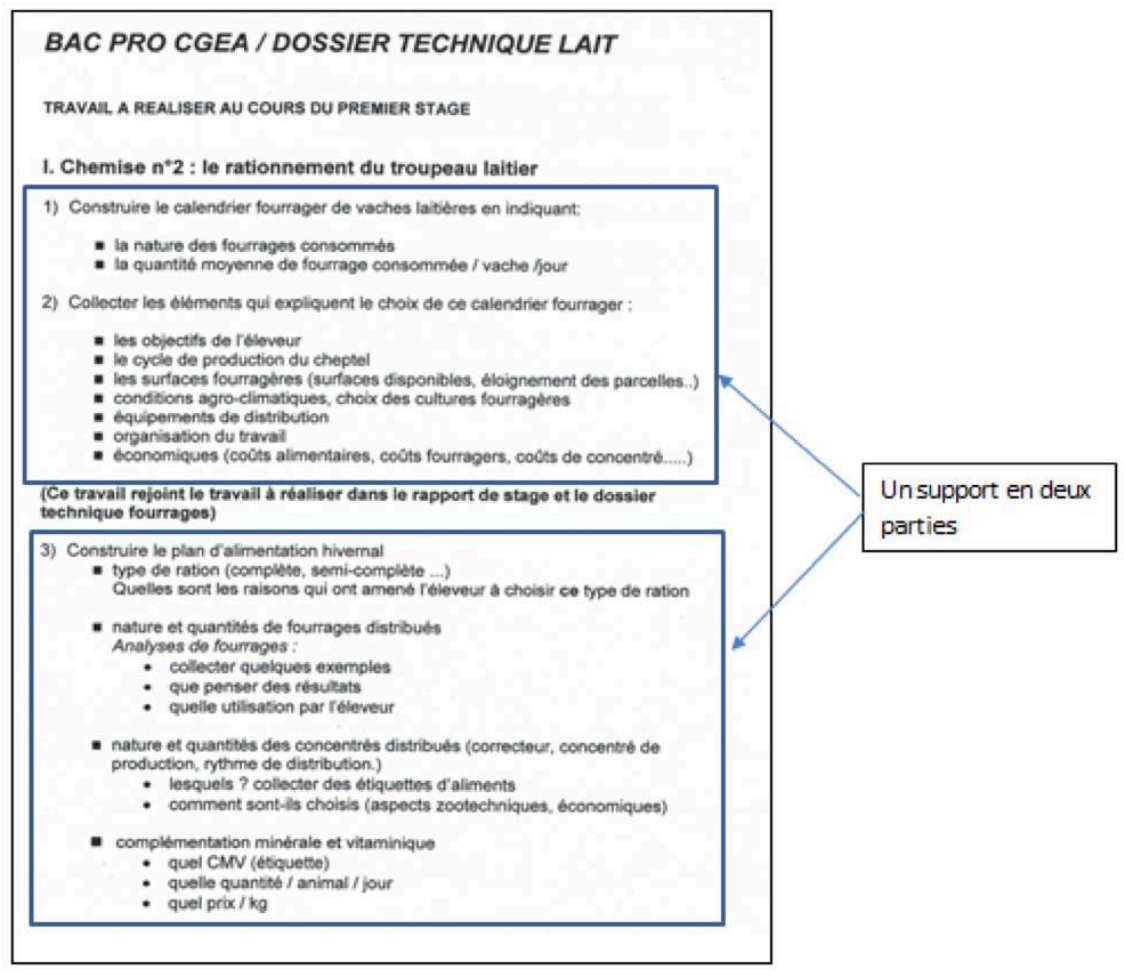

D'un point de vue sémiotique, la centration sur la thématique de l'alimentation partage au moins un aspect avec le support de la MFR, c'est-à-dire une organisation par items. Toutefois ces items sont moins nombreux et sont en relation plus étroite avec la spécificité du thème étudié. Ils ne s'étendent pas à l'ensemble de l'organisation de l'exploitation, contrairement à ce qui constitue la spécificité du support MFR.

En outre, on observe la présence de questions qui sont une aide pour entrer dans une activité de justification, pour mettre en œuvre des écrits analytiques, argumentatifs, ou encore justificatifs («Quelles sont les raisons qui ont amené l'éleveur à choisir ce type de ration?", «que penser des résultats?", «comment (les concentrés) sont-ils choisis?»). Ce n'était pas le cas dans le support précédent davantage fondé sur le renseignement d'indicateurs.

Au lycée trois exercices sont présents dans le support et s'emboîtent en allant du plus simple au plus complexe. Le premier consiste à mettre en rapport les types de culture avec la ration distribuée et ses effets potentiels sur la production laitière en procédant à l'évaluation entre ration et couverture des besoins alimentaires des animaux. Cette interprétation se double de la prise en compte des effets de la ration sur la santé et le métabolisme du troupeau. Enfin, il est demandé de comparer les coûts que représente le poste de l'alimentation sur l'exploitation du stage en s'aidant de moyennes d'exploitation pour objectiver des résultats ainsi que des pratiques, et se faisant d'indiquer d'éventuelles singularités d'exploitation, de réaliser un état des lieux sur les plans techniques et économiques. Ces différentes attentes (recherche d'informationhypothèses-résultats-interprétations) forment un schéma qui relève d'une démarche de raisonnement, voire d'une démarche scientifique. En ce sens, le lycée favorise, par une approche littératiée du travail, une structuration de la pensée en correspondance avec la figure de l'ingénieur agronome. 


\section{Conclusion - Oralité et littératies : Quelques pistes de réflexion}

fabrication des attitudes et des attentes, de formes de discrimination de certains savoirs, de modes de raisonnements par la fréquentation d'un univers ancré dans la littératie ou l'oralité. En effet, les manières de valoriser l'un ou l'autre de ces univers dans un dispositif pédagogique oriente, voire engendre des manières de se rapporter à l'école, au travail et au monde.

31 Ces premières conclusions suggèrent que les apports des notions d'oralité et littératie sont particulièrement heuristiques pour rendre visibles de tels événements. Une des questions en suspens reste celle du continuum entre oralité et littératie que pourrait offrir le discours pédagogique dans l'aller-retour entre support pédagogique et interaction avec les élèves.

Une deuxième conclusion relève des types d'élèves et des pédagogies mises en œuvre. Alors que les plus éloignés de l'école font face à des supports où la dominante est l'oralité, c'est-à-dire où tout le métier est introduit avec toute sa complexité, à l'inverse, les élèves proches des normes scolaires font face à des univers littératiés introductifs à la science appliquée, où le réel est simplifié et modélisé. Ces dimensions qui se conjuguent peuvent plus ou moins jouer en faveur ou en défaveur des élèves. Elles questionnent le principe même d'évitement des codes scolaires pour intéresser les élèves qui en sont les plus lointains. En effet, dans le cas de la MFR l'enseignement repose sur l'oralité du métier sans véritablement interroger les connaissances que les élèves en ont construites, du moins on ne l'a pas observé durant l'année de l'enquête. Sachant qu'une grande partie d'entre eux entrent pour la première fois en contact avec le domaine de l'agriculture par le biais du stage, leurs connaissances sont probablement très faibles et grand est le risque qu'ils se perdent.

Enfin, un des apports de cette étude est de mettre au jour des gradations de pratiques pédagogiques hétérogènes. Parallèle peut être fait avec de nombreux travaux de sociologie des inégalités dans l'enseignement général. Ici l'analyse porte sur un segment scolaire peu analysé dans ses composantes pédagogiques et didactiques, moins encore dans ses composantes écrites peut-être du fait qu'une fois franchi le seuil de l'enseignement professionnel, technique ou agricole ces derniers sont considérés comme sociologiquement neutres et/ou que les outils pour produire des analyses relatives aux enseignements de ce type sont encore largement à composer. Les principales conclusions suggèrent donc qu'il faudrait privilégier les analyses comparatives entre différentes échelles (collège-lycée) de ces voies (professionnelletechnologique-agricole-générale) du système éducatif : enfin des échelles temporelles 
pour penser les modes relationnels d'étude alliant pratiques pédagogiques et modes de faire de la part des élèves.

\section{BIBLIOGRAPHIE}

BARTON, D., \& HAMILTON, M. (1998). Local literacies : reading and writing in one community. Londres/ New York : Routledge.

BARTON, D., \& HAMILTON, M. (2010). « La littératie : une pratique sociale ». Langage et société 133, p. 45-62.

BOUTET, J. (2008). La vie verbale au travail. Toulouse : Octarès.

CHARTIER, A.-M., \& RENARD, P. (2000). « Cahiers et classeurs, les supports ordinaires du travail scolaire ». Repères. Recherches en didactique du français langue maternelle 22, p. 135-159.

CLOT, Y. (2008). Travail et pouvoir d'agir. Paris : Presses universitaires de France.

COLE, M. \& SCRIBNER, S., (1973). « Cognitive Consequences of Formal and Informal Education ». Science 82, p. 553-559.

DENIS, B., \& Theret, M. (1994). « Les grands traités de zootechnie et leur conception de cette discipline ». Ethnozootechnie 54, p. 3-24.

GOODY, J. (1979) [1977]. La Raison graphique. La domestication de la pensée sauvage. Trad. de l'anglais par J. Bazin et A. Bensa. Paris : Éditions de Minuit.

GRosjeAn, M., \& LAcoste, M. (1998). «L'oral et l'écrit dans les communications de travail ou les illusions du "tout écrit" ». Sociologie du Travail 40 (4), p. 439-461.

HEATH, S. B., \& STREET, B. V. (2008). « On Ethnography: Approaches to Language and Literacy Research ». New York/Londres : Teachers College Columbia University.

LATOUR, B., \& WOOLGAR, S. (1988) [1986]. La Production des faits scientifiques. Trad. de l'anglais par M. Biezunski. Paris : La Découverte.

LAPARRA, M., \& MARGOLINAS, C. (2012). « Oralité, littératie et production des inégalités, scolaires ». Le Français Aujourd'hui 177, p. 55-64.

MINASSIAN, L. (2015). « Unification des filières de l'enseignement agricole et diversité d'établissements : un effet positif en termes de réduction des inégalités ? ». Éducation et Sociétés 35, p. 133-150.

TAYLOR, D. (1983). Family Literacy. Young Children Learning to Read and Write. Exeter : Heinemann educational books.

\section{RÉSUMÉS}

L'enseignement agricole a peu fait l'objet de recherches en sociologie. La rareté des travaux a conduit à faire du terrain d'enquête, le fondement d'une élaboration théorique, en particulier 
dans les domaines de la littératie et de l'oralité. L'article proposé repose sur deux études de cas et s'intéresse à l'interpénétration de l'oralité et de la littératie dans deux classes de même niveau, mais de publics différents. Ce parti pris rend visible la manière dont l'agriculture est enseignée à des élèves plus ou moins en difficulté. L'article porte plus précisément sur les supports et le discours pédagogiques en tant que ces derniers peuvent être construits dans une oralité ou littératie plus ou moins grande.

Agricultural education has been little studied in sociology. Because of the scarcity of these studies, we have made it a ground of investigation to found a theoretical elaboration, particularly in the areas of literaty and orality. The proposed article is based on two case studies and focuses on the interpenetration of orality and literacy in two classes on the same level, but with different audiences. This bias makes visible the way in which agriculture is taught to students more or less in scolar difficulty. More specifically, the article focuses on teaching aids and discourse, which can be constructed in a greater or lesser orality or literacy.

\section{INDEX}

Keywords : litteraty, orality, teaching aids, scolar audiences, agricultural education

Mots-clés : littératies, oralité, supports pédagogiques, publics scolaires, enseignement agricole 